\title{
Reinforcement Learning Fuzzy Neural Network Control for Magnetic Shape Memory Alloy Actuator
}

\author{
Miaolei Zhou, Bing Hu, Wei Gao and Jingyuan Wang \\ College of Communication Engineering, Jilin University, Changchun 130022, China \\ zml@jlu.edu.cn; hubing12@mails.jlu.edu.cn; gaow@jlu.edu.cn; \\ jingyuan11@mails.jlu.edu.cn
}

\begin{abstract}
Magnetic shape memory alloy actuator is a new type of actuator that can offer big travel and high resolution of output displacement, which makes it suitable for driving task. However, its output displacement represents the hysteresis applied to input magnetic field. Hysteresis restricts its application in the high precision positioning. In order to eliminate the hysteresis of magnetic shape memory alloy actuator, a reinforcement learning fuzzy neural network controller is proposed. Network structure and special learning algorithm of reinforcement learning fuzzy neural network controller are introduced in detail. The proposed control system adopts the generalized approximate reasoning-based intelligent control architecture, which is mainly consisted of three parts: Action Selection Network, Action Evaluation Network and Stochastic Action Modifier. Finally, in order to verify the effectiveness of the proposed control method, the simulation experiment is researched. The experimental results show that the proposed control method can obtain the smaller tracking error, and the controller's maximum tracking error is less than 0.95\%, hysteresis loop is less than $2.66 \%$.
\end{abstract}

Keywords: magnetic shape memory alloy; hysteresis; reinforcement learning; fuzzy neural network

\section{Introduction}

With the rapid development of precision positioning, micro robot and micro drive field, the traditional actuators are difficult to meet the needs of those fields. Therefore, manufacturing of actuators which make use of new intelligent materials is an urgent demand. Among many new intelligent materials, Magnetic Shape Memory Alloy (MSMA) material is the one which has the characteristics of rapid frequency response and high control precision; all of these include the advantages of both piezoceramic material and giant magnetostrictive material. MSMA material is also able to overcome the disadvantages of low deformation rate of piezoceramic material and giant magnetostrictive material. MSMA actuator exhibits strong hysteresis effect in the output displacement response (see in Figure 1) [1, 2], as is the same as piezoelectric and magnetostrictive material. Due to the hysteresis effect, the positioning accuracy of MSMA actuator is very bad. In order to improve the positioning accuracy of MSMA actuator, the control method needs to be researched [3]. 


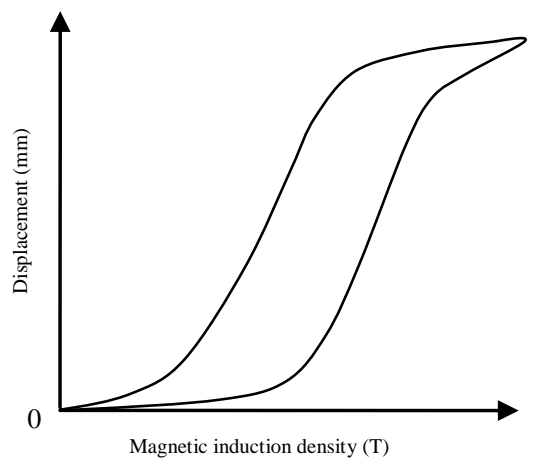

Figure 1. Hysteresis effect of output displacement response of MSMA

The most common control method for minimizing hysteresis nonlinearity is to establish hysteresis model. Hysteresis model mainly has the following categories: (a) Preisach model. Its basic idea is to use many lag elements with simple shape to simulate the complex hysteresis cures [4-6]. It can be applied to many general nonlinearity physical processes with hysteretic characteristics. Preisach model needs a lot of calculation, and a lot of experimental data are needed to estimate parameters of the model. Therefore, Preisach model cannot meet the requirements in cases of requiring high real-time or lacking experimental data. (b) Prandtl-Ishlinskii (PI) model. Its basic idea is that the hysteresis transmission model can be expressed by the superposition of some basic hysteretic operator weights [7, 8]. As a submodel of Preisach model, it has simple structure and less parameter, so it does not need a lot of calculation. (c) Krasnosel'skii-Pokrovskii (KP) model. It is based on the generics play type kernel, and has the similar characters as the Presiach model $[9,10]$. The basic idea lies in the modeling of the real hysteretic transfer characteristic by a weighted superposition of many elementary hysteresis operators, which differ according to the type of the elementary operator in one or more parameters. KP model can work well for off-line identification. Compared with other operator models of hysteresis, KP model has the advantages such as continuity and convergence properties, and therefore, KP model has the ability of modeling complex hysteresis nonlinearities. (d) Duhem model. It can describe the hysteresis with differential equations, which uses the section exponential curve superposition method to approach the hysteresis [11, 12]. Compared with operator hysteresis model, Duhem model changes its output motion direction by changing its input motion direction. Duhem model has the advantage of simple calculation, and Duhem model can select different input functions to characterize more categories of hysteresis characteristic, therefore, Duhem model can deal with any shape of the load-deformation curves.

The modeling methods of the hysteretic nonlinear inverse model above are open loop control methods. Because of modeling errors and disturbance, the precision of open loop control is not very high. To further improve the control precision, some researchers have begun the research by close loop control method.

$\mathrm{Ru}$ et al. proposed a control method for piezoelectric actuator based on a PID feedback controller with a feedforward compensator [13]. A new mathematical model was used to describe the hysteresis phenomenon of a piezoelectric actuator. The experimental results indicate that, in comparison to the regular PID controller, the proposed method has better time and frequency tracking characteristics, and higher positioning accuracy. Chen et al. proposed a tracking control method based on the combination of feedforward and feedback loops for improving the tracking control accuracy of piezoelectric actuators [14]. The feedforward 
controller is established with an inverse hysteresis model based on a neural network, and the hysteresis of piezoelectric actuators is linearized by use of this inverse model. The feedback loop is used to reduce the accumulated error produced by the inverse hysteresis model. The experiment results prove that the proposed controller is effective in improving the tracking accuracy. Xu et al. proposed the inverse modified Prandtl-Ishlinskii model-based feedforward in combination with a PID feedback control algorithm [15]; the feedforward control is used to reduce the effect of the hysteresis nonlinearity, and the PID feedback control is used for the further improving of the control accuracy. The experiment results show that this control algorithm can get good control accuracy in the tracking control of the piezoactuated micropositioning stage. Kyoung et al. proposed a hybrid control method based on Preisach model feed forward control and PID feedback control [16], PID parameters were adjusted by genetic algorithm and fuzzy control algorithm. The experimental results show that the hybrid control algorithm can improve the control performance and reduce the hysteresis effect. Zhou et al. proposed a control method combined with inverse model feedforward control and neural network feedback control, and it has been applied into the hysteresis nonlinearity control of MSMA actuator [17]. In contrast with the proposed feedforward control method, the proposed hybrid control method can improve the accuracy of the output displacement by $133.98 \%$. Gu et al. proposed a hybrid control strategy combining a model-based feedforward controller and a PID feedback loop for high-accuracy and high-speed tracking control of piezoelectric actuators [18]. A feedforward controller is developed to cancel the rate-dependent hysteresis based on the inverse ellipse-based mathematic model, while the PID controller is used to compensate for the creep, modeling errors, and parameter uncertainties. The experimental results show that hybrid controller can reduce the root-mean-square tracking error to only $0.34 \%$ of the displacement range under the input frequency of $100 \mathrm{~Hz}$. Jiang et al. proposed a new asymmetric hysteretic nonlinear modeling method for the piezoelectric actuator [19]. This method uses the two asymmetric hysteretic operators to establish the model respectively for the rising edge and the falling edge of the hysteretic loop, in addition, the use of the recursive least square simplifies the calculation of the model. Through the comparison of the experimental data, it has been proved that the asymmetric hysteretic nonlinear model has a high accuracy.

In this paper, a reinforcement learning fuzzy neural network control method is proposed to improve output displacement's precision of MSMA actuator. The proposed controller adopts generalized approximate reasoning-based intelligent control (GARIC) architecture. GARIC is consisted of three parts: the Action Selection Network (ASN), the Action Evaluation Network (AEN) and the Stochastic Action Modifier (SAM). Special structure and design of every part is described in detail. Finally, the simulation experiment is researched according to the proposed control method.

\section{Design of Reinforcement Learning Fuzzy Neural Network Controller}

The proposed reinforcement learning fuzzy neural network controller adopts GARIC architecture (See in Figure 2) [20]. In Figure 2, $x$ is the current system state, $r$ is an external excitation signal, when the system fails, its value is 1 , otherwise its value is 0 , and $f^{\prime}$ is the output control value which is to make $r$ as small as possible. AEN is state estimator whose function is, according to the state of the system $x$, to evaluate the system operation state so as to generate the estimation value $v$ of external reinforcement signal, according to $x$ and $v$ produce internal reinforcement signal $\hat{r}$.

The whole system will accord to $\hat{r}$ to revise the parameters of AEN and ASN, and achieve the required control precision. The role of ASN is to generate the recommended control value 
of $f$. The role of the SAM is to combine internal reinforcement signal and recommended control value to produce a best control value $f^{\prime}$.

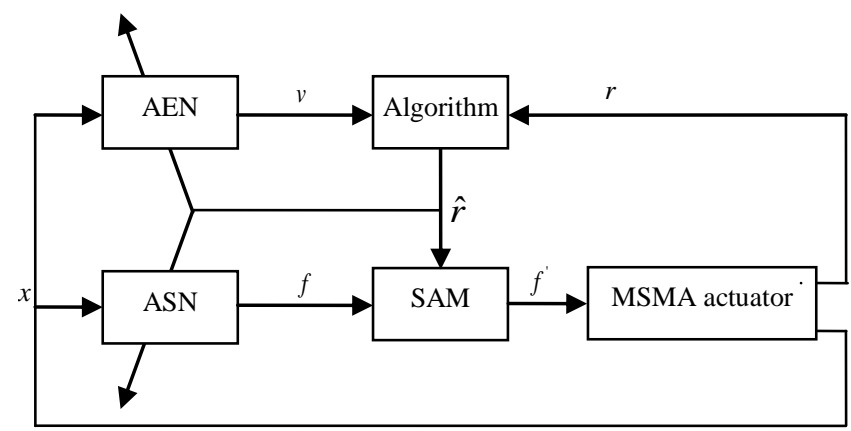

Figure 2. The system structure of generalized approximate reasoning-based intelligent control (GARIC)

\subsection{Structure of AEN}

The structure of AEN is a three-layer neural network. The inputs of this neural network are $x$ and $x^{\prime} . x$ is the error between given signal and actual output signal, $x^{\prime}$ is the change of error between given signal and actual output signal and $v$ is the output of AEN and the estimation value of current system state.

Specific structure of AEN is shown in Figure 3. $a_{i j}$ is weight coefficient between input layer and hidden layer, $b_{i}$ is weight coefficient between the input layer and the output layer, and $c_{j}$ is weight coefficient between the hidden layer and the output layer, where $i=1,2, \quad j=1,2, \ldots, 16$. The special work principle of every layer is as follows:

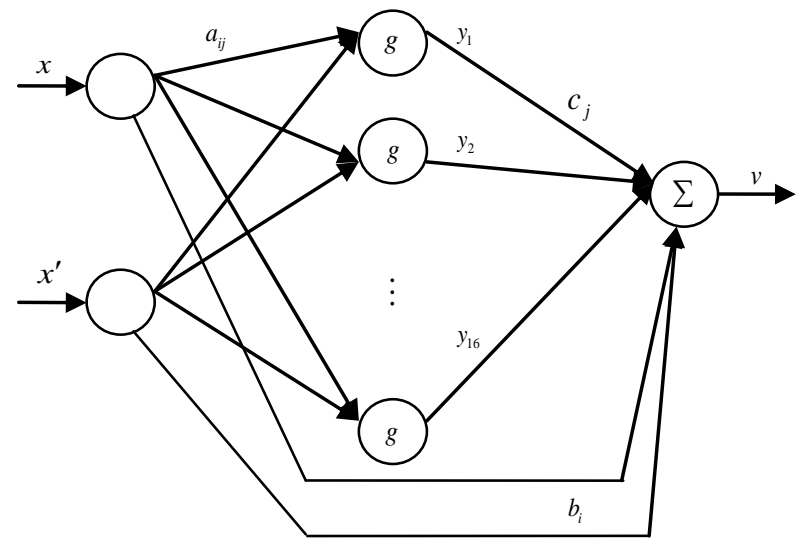

Figure 3. The structure of AEN

1) Input layer.

The number of neurons in the input layer is 2. Input values of two neurons are respectively $x$ and $x^{\prime}$ that can describe the current state of the MSMA, and the input layer can be 
described as

$$
n e t^{1}=\left[\begin{array}{ll}
x & x^{\prime}
\end{array}\right]
$$

2) Hidden layer.

The number of neurons in the hidden layer is 16 . The hidden layer receives signals from the input layer, and the signals can be described as

$$
\begin{gathered}
y_{j}(t, t+1)=g\left[\sum_{i=1}^{2} a_{i j}(t) \cdot x_{i}(t+1)\right] \\
g(s)=\frac{1}{1+\exp (-s)}
\end{gathered}
$$

where $y_{j}(t, t+1)$ have two time variables. The significance of time variables is to emphasize the weigh value at moment $t$ and the input signal at moment $t+1$.

3) Output layer.

The number of neurons in the output layer is 1 . The input values in the output layer are signals from the input layer and the hidden layer, and the output value is state estimation signal $v$. In the output layer, output signal is linearized into

$$
v(t, t+1)=\sum_{i=1}^{2} b_{i}(t) x_{i}(t+1)+\sum_{j=1}^{16} c_{j}(t) y_{j}(t, t+1)
$$

\subsection{Algorithm of Internal Reinforcement Signal}

Internal reinforcement signal $\hat{r}$ is based on the output state estimation signal $v$ from AEN and external reinforcement signal $r$ to generate, the algorithm is as follows

$$
\hat{r}(t+1)=\left\{\begin{array}{cc}
0 & \text { initial state } \\
r(t+1)-v(t, t) & \text { error state } \\
\delta v(t, t+1)-v(t, t) & \text { other state }
\end{array}\right.
$$

where $\delta$ is discount rate $(0 \leq \delta \leq 1) . \delta$ can make the action of $v(t, t)$ less than $v(t, t+1)$. $r(t+1)$ is a signal at moment $t+1$, its value is -1 when error occurs, else is 0 .

The values of $v(t, t)$ and $v(t, t+1)$ are in the $[-1,0]$. When error occurs, value of $r(t+1)$ is -1 , value of $\hat{r}(t+1)$ is in the $[-1,0],-1$ indicates that the prediction is error and needs to be punished, 0 indicates that the prediction is correct and does not need to be modified. When no error occurs, value of $r(t+1)$ is 0 , value of $\hat{r}(t+1)$ is in the [-1,1], -1 expresses the system state from right to error and should be punished; and 1 expresses the system state from error to right and should be rewarded. Finally, the generated internal reinforcement signal $\hat{r}(t+1)$ will be used to learn the AEN and ASN.

\subsection{Structure of ASN Fuzzy Neural Network}

ASN is equivalent to a fuzzy controller with the neural network form [21, 22]. In order to simplify the complexity of the controller, ASN fuzzy neural network, as a four layer fuzzy neural network, adopts the Gauss function as membership function. The network structure is 
shown in Figure 4.

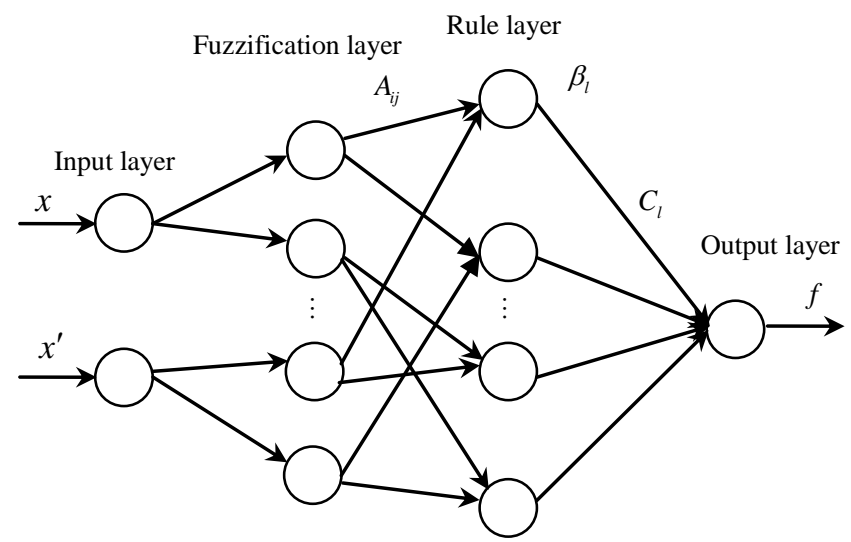

Figure 4. Network structure of ASN

1) Input layer.

The number of neurons in the input layer is 2, where $x$ and $x^{\prime}$ are error and change of error respectively, input layer can be described as

$$
n e t^{1}=\left[\begin{array}{ll}
x & x^{\prime}
\end{array}\right]
$$

2) Fuzzification layer.

The number of neurons in the fuzzification layer is 10. Input of system can be defined as five fuzzy sets that are negative big (NB), negative small (NS), zero (Z), positive small (PS), positive big (PB) respectively. Serving as input membership function, Gauss function is used to calculate the corresponding membership function value.

$$
\begin{gathered}
n e t^{2}=-\frac{\left(x_{i}-m_{i j}\right)^{2}}{\sigma_{i j}^{2}} \\
A_{i j}=\exp \left(n e t^{2}\right)
\end{gathered}
$$

In equation (7), $m_{i j}$ and $\sigma_{i j}$ are respectively the mean and standard deviation of Gauss function of the $j$-th fuzzy set of the $j-$ th input variables, and are the adjustable parameters. In addition, $i=1,2, \quad j=1,2, \ldots, 5$.

3) Rule layer.

The number of neurons in the rule layer is 25 . The input is membership function value of each corresponding fuzzy sets, the output is the activation degree of the corresponding rules.

Fuzzy rule is:

$$
R_{1}: \quad \text { If } x_{1}=A_{1}^{l} \text { and } x_{2}=A_{2}^{l} \text {, Then } y=C_{l}
$$

where, $l=1,2, \ldots, 25$ represents the number of rules, $C_{l}$ is weigh value of the corresponding activation degree. In the paper, multiplication is adopted as operation form to 
simply calculation, namely

$$
\beta_{l}=A_{1}^{l} A_{2}^{l}
$$

where, $\beta_{l}$ represents the activation degree of the $l-t h$ rule.

4) Output layer.

The number of neurons in the output layer is 1 . The output is described as

$$
f=K_{l} \cdot\left(\sum_{i=1}^{l} \beta_{l} C_{l} / \sum_{i=1}^{l} \beta_{l}\right)
$$

where $f$ is output of the ASN. $K_{l}$ is scale factor, $K_{l}=0.15$ in the paper. $C_{l}$ is fuzzy rule table of the controller, can be used as adjustable weight coefficients between the third layer and the fourth layer. Initial weigh values of $C_{l}$ are shown in Table 1.

Table 1. Fuzzy rule control table

\begin{tabular}{|c|c|c|c|c|c|}
\hline $\begin{array}{c}x^{\prime} \\
x\end{array}$ & $\mathrm{NL}$ & $\mathrm{NS}$ & $\mathrm{Z}$ & $\mathrm{PS}$ & $\mathrm{PL}$ \\
\hline $\mathrm{NL}$ & 4 & 3 & 2 & 1 & 0 \\
\hline $\mathrm{NS}$ & 3 & 2 & 1 & 0 & -1 \\
\hline $\mathrm{Z}$ & 2 & 1 & 0 & -1 & -2 \\
\hline $\mathrm{PS}$ & 1 & 0 & -1 & -2 & -3 \\
\hline $\mathrm{PL}$ & 0 & 1 & -2 & -3 & -4 \\
\hline
\end{tabular}

\subsection{Stochastic Action Modifier (SAM)}

Gradient descent algorithm is widely used to learn the network. But it is easy for this method to make the control variable fall into local minimum value so that the whole system has steady state error. In order to prevent the control variable from falling into local minimum value, the control signal adopts the SAM.

The learning algorithm is described as

$$
s(t)=\left\{\begin{array}{cl}
f^{\prime} & |x| \leq x_{e} \\
\operatorname{SAM}(f) & |x|>x_{e}
\end{array}\right.
$$

where $x_{e}$ is the preset control precision of the controller.

The meaning of equation (11) is: when the actuator output does not exceed the preset control precision, in order to obtain steady state, it does not adopt SAM method; and when the actuator output exceeds the preset control precision, it adopts the SAM to control the signal.

SAM method randomly generates a control variable $\hat{f}$ based on the recommended control variable $f^{\prime}$ and internal reinforcement signal $\hat{r} . \hat{f}$ meets the Gaussian distribution whose mean is $f^{\prime}$ and standard deviation is $e^{-t} \sigma(\hat{r}(t-1))$.

$\sigma()$ adopts a monotone non-negative function $\exp (-\hat{r})$. Furthermore, the purpose of introducing $e^{-t}$ is to make the perturbation amplitude decrease over time and improve control 
accuracy. The normalized deviation of recommended control variable and actual control variable is

$$
s(t)=\frac{\hat{f}(t)-f^{\prime}(t)}{\sigma(\hat{r}(t-1))}
$$

$s(t)$ is learning factor of ASN.

\section{Learning Algorithm of AEN and ASN}

\subsection{Learning of AEN}

AEN is similar to the reward learning rules. If internal reinforcement signal is positive, corresponding weigh value is rewarded. Conversely, if internal reinforcement signal is negative, corresponding weigh value is punished. The learning algorithm of weigh value $b_{i}$ and $c_{j}$ are described as

$$
\begin{gathered}
b_{i}(t+1)=b_{i}(t)+\eta_{\mathrm{AEN}} \hat{r}(t+1) x(t) \\
c_{j}(t+1)=c_{j}(t)+\eta_{\mathrm{AEN}} \hat{r}(t+1) y_{j}(t)
\end{gathered}
$$

where $\eta_{A E N}$ is learning rate.

Algorithm of the weigh value between the input layer and the output layer adopts the modified BP algorithm.

$$
a_{i j}(t+1)=a_{i j}(t)+\eta_{\mathrm{AEN}} \hat{r}(t+1) y_{j}(t, t)\left(1-y_{j}(t, t)\right) \operatorname{sgn}\left[c_{j}(t)\right] x_{i}(t)
$$

In the above weight learning algorithm, the purpose of substitute $c_{i}(t)$ with $\operatorname{sgn}\left(c_{i}(t)\right)$ is to enhance the robustness of the algorithm.

\subsection{Learning of ASN}

To be learning parameters of ASN are respectively membership parameters $m_{i j}$ and $\sigma_{j}$ of the input variable, and weight value $C_{l}$ in the rule layer. $p$ is used to represent the parameters that need to be learned. The purpose of ASN is to make $v$ biggest by adjusting the parameter $p$ and improve the performance of the system.

The parameter is adjusted by using gradient learning method, namely,

$$
\Delta p=\eta_{\mathrm{ASN}} s(t) \hat{r}(t) \frac{\partial v}{\partial p}=\eta_{\mathrm{ASN}} s(t) \hat{r}(t) \frac{\partial v}{\partial f^{\prime}} \frac{\partial f^{\prime}}{\partial p}
$$

In the equation (16), $\hat{r}(t)$ and $s(t)$ are used as learning signal. The purpose is that if a large perturbation can obtain good control effect, this perturbation will obtain more rewards, conversely, if the larger perturbation can not achieve good results, it will reduce the impact on weight.

Since the relation between $f^{\prime}$ and $v$ is unknown and difficult to calculate, we use the instantaneous difference ratio with $f^{\prime}$ and $v$ replaces $\partial v / \partial f^{\prime}$, so there is 


$$
\frac{\partial v}{\partial f^{\prime}} \approx \frac{d v}{d f^{\prime}} \approx \frac{v(t)-v(t-1)}{f^{\prime}(t)-f^{\prime}(t-1)}
$$

As equation (17) is only cursory approximation calculation, its sign is used and its value is not used in the paper.

The calculation of the second item $\partial f^{\prime} / \partial p$ in equation (16) is relatively simple, there into computational formula of $C_{l}(l=1,2, \ldots, 25)$.

$$
\frac{\partial f^{\prime}}{\partial C_{l}}=\partial\left(\sum_{l=1}^{25} \beta_{l} C_{l} / \sum_{l=1}^{25} \beta_{l}\right) / \partial C_{l}=\beta_{l} / \sum_{l=1}^{25} \beta_{l}
$$

By equations (16) and (17), the recurrence formula of $C_{l}$ can be described as

$$
C_{l}(t+1)=C_{l}(t)+\eta_{\mathrm{AEN}} s(t) \hat{r}(t) \operatorname{sgn}\left(\frac{v(t)-v(t-1)}{f^{\prime}(t)-f^{\prime}(t-1)}\right)\left(\beta_{l} / \sum_{l=1}^{25} \beta_{l}\right)
$$

Calculation rules for mean $m_{i j}$ and standard deviation $\sigma_{i j}$ of membership function.

Based on equations (16) and (17), the calculation formula is as follows

$$
\frac{\partial f^{\prime}}{\partial p}=\frac{\partial f^{\prime}}{\partial A_{n}^{l}} \frac{\partial A_{n}^{l}}{\partial p}=\left(\sum_{A_{n}^{l}} \frac{\partial f^{\prime}}{\partial \beta_{l}} \frac{\partial \beta_{l}}{\partial A_{n}^{l}}\right) \frac{\partial A_{n}^{l}}{\partial p}
$$

where

$$
\begin{aligned}
\frac{\partial f^{\prime}}{\partial \beta_{l}}=\left(C_{l} \sum_{l=1}^{25} \beta_{l}-\sum_{l=1}^{25} \beta_{l} C_{l}\right) /\left(\sum_{l=1}^{25} \beta_{l}\right)^{2}=\left(C_{l}-f^{\prime}\right) / \sum_{l=1}^{25} \beta_{l} \\
\frac{\partial \beta_{l}}{\partial A_{1}^{l}}=A_{2}^{l} \\
\frac{\partial \beta_{l}}{\partial A_{2}^{l}}=A_{1}^{l}
\end{aligned}
$$

General equations (20), (21), (22) and (23) can deduce that the learning rule of mean $m_{i j}$ and standard deviation $\sigma_{i j}$ in the fuzzification layer is

$$
\begin{aligned}
& m_{i j}(t+1)=m_{i j}(t)+\eta_{\mathrm{ASN}} s(t) \hat{r}(t) \operatorname{sgn}\left(\frac{v(t)-v(t-1)}{f^{\prime}(t)-f^{\prime}(t-1)}\right) \frac{\partial f^{\prime}(t)}{\partial A_{n}^{l}} \exp \left(-\frac{\left(x-m_{i j k}\right)^{2}}{\sigma_{i j}^{2}}\right)\left(\frac{2\left(x-m_{i j}\right)}{\sigma_{i j}^{2}}\right) \\
& \sigma_{i j}(t+1)=\sigma_{i j}(t)+\eta_{\mathrm{ASN}} s(t) \hat{r}(t) \operatorname{sgn}\left(\frac{v(t)-v(t-1)}{f^{\prime}(t)-f^{\prime}(t-1)}\right) \frac{\partial f^{\prime}(t)}{\partial A_{n}^{l}} \exp \left(-\frac{\left(x-m_{i j k}\right)^{2}}{\sigma_{i j k}^{2}}\right)\left(\frac{2\left(x-m_{i j k}\right)^{2}}{\sigma_{i j k}^{3}}\right)
\end{aligned}
$$

\section{Simulation Research}

In order to verify the performance of the proposed control algorithm, simulation experiment was investigated in the paper.

The initial values of parameters and variables in the simulation are shown in Table 2. 
Table 2. The initial value of some parameters and variable in the experiment

\begin{tabular}{|c|c|c|c|}
\hline $\begin{array}{l}\text { Sym } \\
\text { bol }\end{array}$ & Quantity & Type & Value \\
\hline$a$ & $\begin{array}{l}\text { input layer to hidden layer } \\
\text { weights of AEN }\end{array}$ & $\begin{array}{l}2 \times 16 \\
\text { matrix }\end{array}$ & $\begin{array}{l}\text { random number in }[-0.1, \\
0.1]\end{array}$ \\
\hline$b$ & $\begin{array}{l}\text { input layer to output layer } \\
\text { weights of AEN }\end{array}$ & $1 \times 2$ matrix & $\begin{array}{l}\text { random number in }[-0.1 \text {, } \\
0.1]\end{array}$ \\
\hline$d$ & $\begin{array}{l}\text { hidden layer to output layer } \\
\text { weights of AEN }\end{array}$ & $\begin{array}{l}1 \times 16 \\
\text { matrix }\end{array}$ & $\begin{array}{l}\text { random number in }[-0.1, \\
0.1]\end{array}$ \\
\hline$\hat{r}_{0}$ & $\begin{array}{l}\text { initial value of internal } \\
\text { reinforcement signal }\end{array}$ & constant & 0 \\
\hline$\delta$ & $\begin{array}{l}\text { discount rate of internal } \\
\text { reinforcement for } v(t, t+1)\end{array}$ & constant & 0.9 \\
\hline$m_{j}$ & $\begin{array}{c}\text { initial mean value of } \\
\text { displacement membership } \\
\text { function in the fuzzification } \\
\text { layer of ASN }\end{array}$ & $1 \times 5$ matrix & {$[-1.0,-0.5,0,0.5,1.0]$} \\
\hline$\sigma_{j}$ & $\begin{array}{c}\text { initial variance value of } \\
\text { displacement membership } \\
\text { function in the fuzzification } \\
\text { layer of ASN }\end{array}$ & $1 \times 5$ matrix & {$[0.3,0.3,0.3,0.3,0.3]$} \\
\hline$m_{j}^{\prime}$ & $\begin{array}{l}\text { initial mean value of velocity } \\
\text { membership function in the } \\
\text { fuzzification layer of ASN }\end{array}$ & $1 \times 5$ matrix & {$[-3.0,-1.5,0,1.5,3.0]$} \\
\hline$\sigma_{j}^{\prime}$ & $\begin{array}{c}\text { initial variance value of } \\
\text { velocity membership function } \\
\text { in the fuzzification layer of } \\
\text { ASN }\end{array}$ & $1 \times 5$ matrix & {$[1.0,1.0,1.0,1.0,1.0]$} \\
\hline$C_{l}$ & $\begin{array}{l}\text { initial weight value of in the } \\
\text { rule layer of ASN }\end{array}$ & $\begin{array}{r}1 \times 25 \\
\text { matrix } \\
\end{array}$ & See in the Table 1 \\
\hline$x_{e}$ & displacement accuracy & constant & 0.01 \\
\hline$x_{e}^{\prime}$ & velocity accuracy & constant & 0.1 \\
\hline$\eta_{\mathrm{AEN}}$ & Learning radio of AEN & constant & 0.1 \\
\hline$\eta_{\mathrm{ASN}}$ & Learning radio of ASN & constant & 0.01 \\
\hline
\end{tabular}

Figure 5 shows the result that the output of MSMA actuator tracks the sinusoidal wave signal. It can be seen that the controller can track the sinusoidal wave signal after a short time of learning. In Figure 5, red broken line is the sinusoidal wave signal and blue real line is actual output displacement curve of MSMA actuator. 


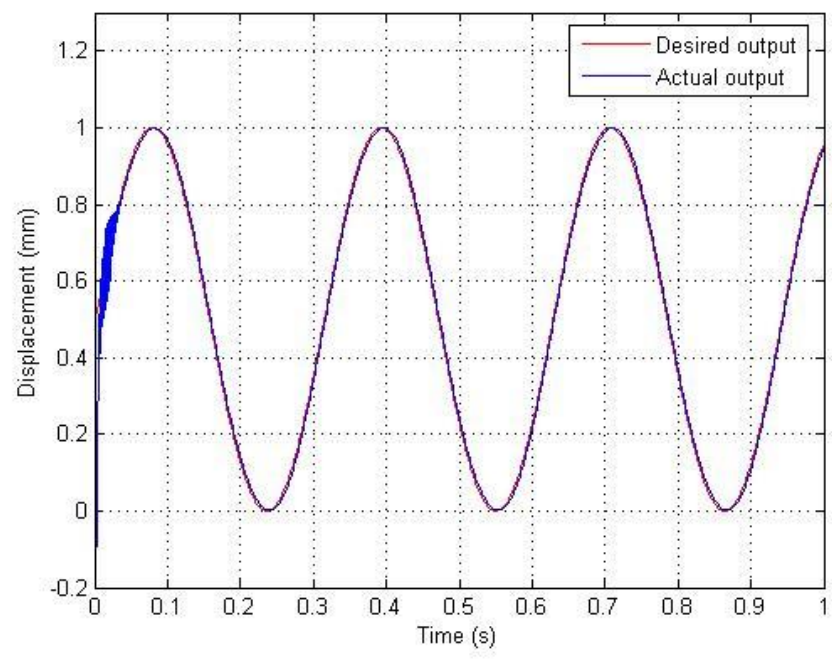

Figure 5. System Response with Respect to Sinusoidal Wave Reference Input

Figure 6 shows the error curve that output of MSMA actuator tracks the sinusoidal wave signal. The maximum tracing error of the proposed control method is less than $0.95 \%$ after a short time of learning. Figure 6 indicates that the proposed control method can obtain more accurate control results for MSMA actuator.

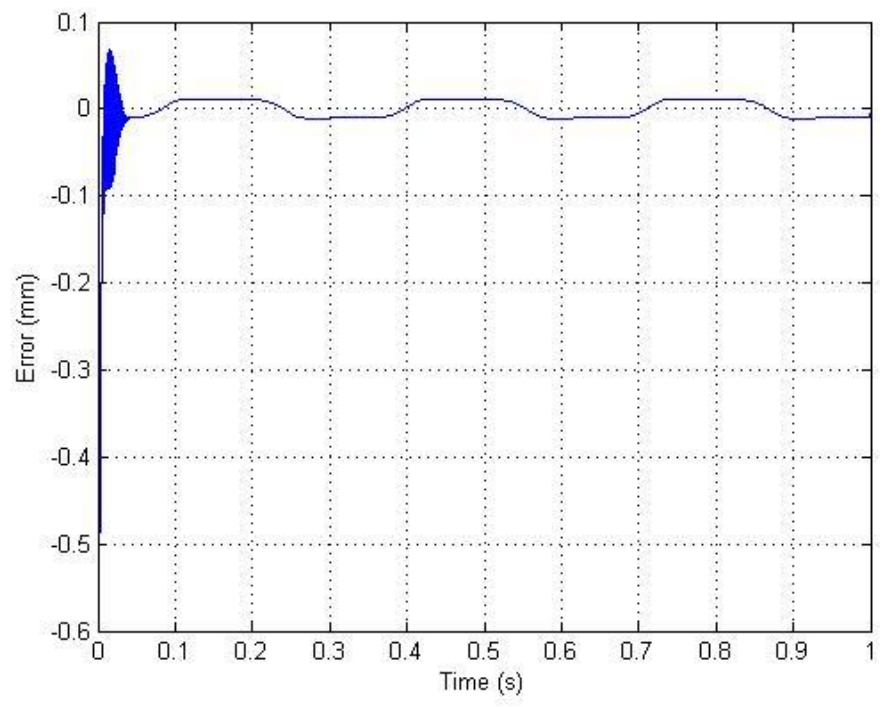

Figure 6. Tracing Error with Respect to Sinusoidal Wave Reference Input

Figure 7 shows the hysteresis loop output curve of the system under the proposed control method. It is calculated that the hysteresis loop is less than $2.66 \%$. The simulation results show the proposed control method can reduce hysteresis phenomenon of MSMA. 


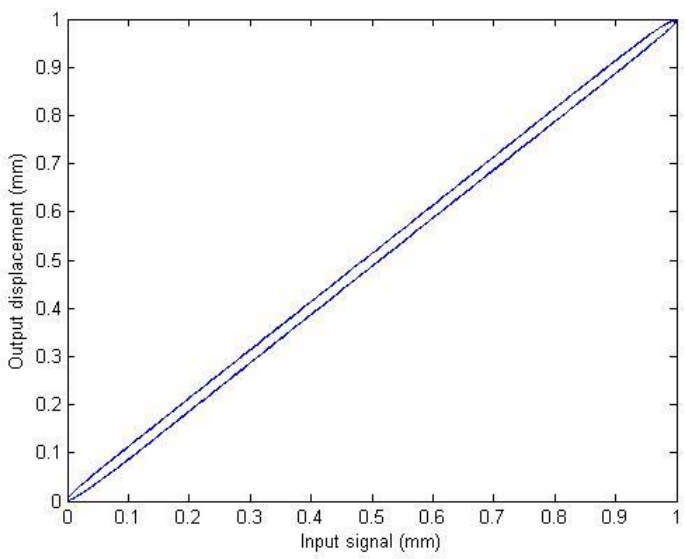

Figure 7. Input/Output Relation Curve under the Proposed Control Method

\section{Conclusions}

The hysteresis limits the further application of MSMA actuator. Currently the research work about its high precision displacement control method has rarely appeared. A reinforcement learning fuzzy neural network control approach is proposed to improve the output displacement's precision of MSMA actuator in the paper. From the foregoing presentation and discussion of the novel RLFNNCMSMAA, main conclusions may be drawn as follows:

- The proposed control approach adopts GARIC architecture. It is mainly consisted of three parts: ASN, AEN and SAM. GARIC learns to control the MSMA actuator by use of reinforcement learning. The fuzzy controller in GARIC learns by tuning its membership functions. The basic structure of AEN is a three-layer neural network, the learning rule adopts rewards and punishment learning rule, the weight algorithm between the input layer and the hidden layer adopts the improved BP algorithm, ASN fuzzy neural network is a four-layer fuzzy neural network and adopts the Gauss membership function, using gradient learning method to adjustment the parameters of system. In order to avoid the control variable to fall into local minimum value, the control signal adopts the SAM. The SAM uses the internal reinforcement to determine an action $f^{\prime}$ that is applied to the plant. $f^{\prime}$ is a Gaussian random variable with mean $\hat{f}$. The standard deviation of $f^{\prime}$ is a function of the internal reinforcement.

- Hysteresis of MSMA actuator can be eliminated by the proposed control method. The experimental results show that the output value can track a given sinusoidal wave signal under the proposed control method, the maximum tracking error is less than $0.95 \%$, and the hysteresis loop is less than $2.66 \%$. It illustrates that the proposed control method can achieve good control effect.

\section{Acknowledgements}

This research was supported in part by the National Natural Science Foundation of China (No. 51105170), and the Program of Science and Technology Development Plan of Jilin province of China (No. 201105015). 


\section{References}

[1] K. Ullakko, "Magnetically controlled shape memory alloys: A new class of actuator materials", Journal of Materials Engineering and Performance, vol. 5, no. 1, (1996), pp. 2170-2183.

[2] T. Krenke, S. Aksoy, E. Duman, M. Acet, X. Moya, L. Mañosa and A. Planes, "Hysteresis effects in the magnetic-field-induced reverse martensitic transition in magnetic shape memory alloys", Journal of Applied Physics, vol. 108, no. 4, (2010), pp. 1-4.

[3] I. Suorsa, J. Tellinen, E. Pagounis, I. Aaltio and K. Ullakko, "Applications of magnetic shape memory actuators", The 8th international conference ACTUATOR, Bremen, Germany, (2002) June 10-12, pp. 158161.

[4] L. Dupré, R. V. Keer and J. Melkebeek, "An iron loss model for electrical machines using the Preisach theory", IEEE Transactions on Magnetics, vol. 33, no. 5, (1997), pp. 4158-4160.

[5] X. B. Tan and S. B. John, "Modeling and control of hysteresis in magnetostrictive actuators", Automatica, vol. 40, no. 9, (2004), pp. 1469-1480.

[6] M. L. Zhou, W. Gao and Y. T. Tian, "Hybrid control based on inverse Prandtl-Ishlinskii model for magnetic shape memory alloy actuator", Journal of Central South University, vol. 20, no. 5, (2013), pp. 1214-1220.

[7] M. C. Deng, C. G. Jiang, A. Inoue and C. Y. Su, "Operator-based robust control for nonlinear systems with Prandtl-Ishlinskii hysteresis", International Journal of Systems Science, vol. 42, no. 4, (2011), pp. 643-652.

[8] C. Y. Su, Q. Q. Wang, X. K. Chen and S. Rakheja, "Adaptive variable structure control of a class of nonlinear systems with unknown Prandtl-Ishlinskii hysteresis", IEEE Transactions on Automatic Control, vol. 50, no. 12, (2005), pp. 2069-2074.

[9] J. Hartmut and K. Klaus, "Real-time compensation of hysteresis and creep in piezoelectric actuators", Sensors and Actuators A: Physical, vol. 79, no. 2, (2000), pp. 83-89.

[10] G. V. Webb, D. C. Lagoudas and A. J. Kurdila, "Hysteresis modeling of SMA actuators for control applications", Journal of Intelligent Material Systems and Structures, vol. 9, no. 6, (1998), pp. 432-448.

[11] I. D. Mayeyergoyz, "Mathamatical Model of Hysteresis", IEEE Transactions on Magnetics, vol. 22, no. 5, (1986), pp. 603-608.

[12] A. Toyohiko and O. Takanobu, "One-dimensional shape memory alloy problem with Duhem type of hysteresis operator", International Series of Numerical Mathematics, vol. 154, (2007), pp. 1-9.

[13] C. H. Ru and L. N. Sun, "Improving positioning accuracy of piezoelectric actuators by feedforward hysteresis compensation based on a new mathematical model", Review of Scientific Instruments, vol. 76, no. 9, (2005), pp. 1-8.

[14] Y. S. Chen, J. H. Qiu, H. L. Ji and K. J. Zhu, "Tracking control of piezoelectric actuator system using inverse hysteresis model”, International Journal of Applied Electromagnetics and Mechanics, vol. 33, no. 3-4, (2010), pp. 1555-1564.

[15] Q. S. Xu and Y. M. Li, "Precise tracking control of a piezoactuated micropositioning stage based on modified Prandtl-Ishlinskii hysteresis model", IEEE Conference on Automation Science and Engineering, Toronto, Canada, (2010) August 21-24, pp. 692-697.

[16] K. A. Kyoung and B. K. Nguyen, "Modeling and control of shape memory alloy actuators using Preisach model, genetic algorithm and fuzzy logic", Mechatronics, vol. 18, no. 3, (2008), pp. 1555-1564.

[17] M. L. Zhou, W. Gao and F. Liu, "Hybrid control method for hysteresis of magnetically controlled shape memory actuator", International Journal of Materials and Product Technology, vol. 44, no. 1, (2012), pp. 77 91.

[18] G. Y. Gu and L. M. Zhu, "High-speed tracking control of piezoelectric actuators using an ellipse-based hysteresis model”, Review of Scientific Instruments, vol. 81, no. 8, (2010), pp. 1-9.

[19] H. Jiang, H. L. Ji, J. H. Qiu and Y. S. Chen, "A modified prandtl-ishlinskii model for modeling asymmetric hysteresis of piezoelectric actuators", IEEE Transactions on Ultrasonics, Ferroelectrics, and Frequency Control, vol. 57, no. 2, (2010), pp. 1200-1210.

[20] H. R. Berenji and P. Khedkar, "Learning and tuning fuzzy logic controllers through reinforcements", IEEE Transactions on Neural Networks, vol. 3, no. 5, (1992), pp. 724-740.

[21] A. Likas, "Reinforcement learning using the stochastic fuzzy min-max neural network", Neural Processing Letters, vol. 13, no. 3, (2001), pp. 213-220.

[22] Y. Duan, Q. Liu and X. H. Xu, "Application of reinforcement learning in robot soccer", Engineering Applications of Artificial Intelligence, vol. 20, no. 7, (2007), pp. 936-950. 


\section{Authors}

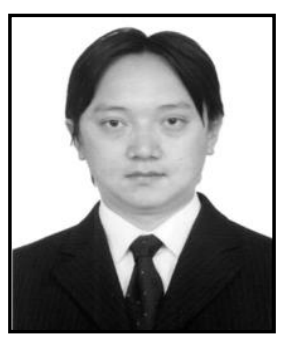

\section{Miaolei Zhou}

He was born in China in 1976. He received his M.E. in control science and engineering (2000) from Jilin Technology of University, China, and PhD in control theory and application (2004) from Jilin University, China. He was a postdoctoral researcher from 2006 to 2008 in Tokyo University, Japan. Now he is an associate professor in the college of communication engineering, Jilin University, China. $\mathrm{He}$ is a member of an editorial board of "Scientific Journal of Control Engineering". His research interests include micro/nano drive and control technology, intelligent control.

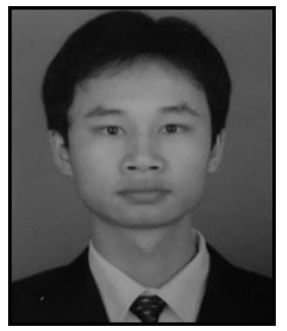

\section{Bing Hu}

He was born in China in 1986. He received his B. S. degree in the mechanical and electrical engineering (2010) from Wuhan Textile University, China. Currently, he is a postgraduate student in the college of communication engineering, Jilin University, China. His recent research interest is neural network control for magnetic shape memory alloy actuator.

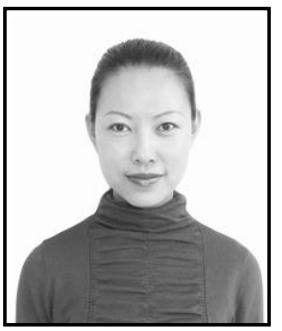

\section{Wei Gao}

She was born in China in 1974. She received her M.E. in control science and engineering (1999) and $\mathrm{PhD}$ in control theory and application (2003) from Jilin University, china. Now she is an associate professor in the college of communication engineering, Jilin University, China. Her current research interests include micro/nano drive and control technology.

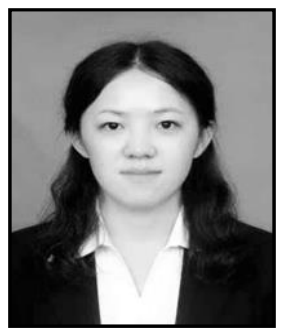

\section{Jingyuan Wang}

She was born in China in 1988. She received her B. S. degree in the electrical and electronic engineering(2011) from Changchun University of Technology, China. Currently, she is a postgraduate student in the college of communication engineering, Jilin University, China. Her recent research interest is intelligent control for piezoelectric actuator. 